\title{
An Adaptation Reference-point-based Multiobjective Evolutionary Algorithm
}

\author{
Juan Zou ${ }^{\mathrm{a}, \mathrm{b}, \mathrm{c}}$, Liuwei Fuc, ${ }^{\mathrm{c}, *}$, Shengxiang Yang ${ }^{\mathrm{e}}$, Jinhua Zheng ${ }^{\mathrm{c}, \mathrm{d}}$, Gan Ruan ${ }^{\mathrm{c}}$, Tingrui Pei ${ }^{\mathrm{a}}$, Lei Wang ${ }^{\mathrm{c}}$ \\ ${ }^{a}$ Key Laboratory of Hunan Province for Internet of Things and Information Security, Xiangtan University, Hunan Province, China \\ ${ }^{b}$ LED Lighting Research \& Technology Center of Guizhou TongRen, GuiZhou, China \\ ${ }^{c}$ Key Laboratory of Intelligent Computing and Information Processing, Ministry of Education, Xiangtan University, Xiangtan, Hunan \\ Province, China \\ ${ }^{d}$ Hunan Provincial Key Laboratory of Intelligent Information Processing and Application, Hengyang, 421002, China \\ ${ }^{e}$ School of Computer Science and Informatics, De Montfort University, Leicester LE1 9BH,U.K.
}

\begin{abstract}
It is well known that maintaining a good balance between convergence and diversity is crucial to the performance of multiobjective optimization algorithms (MOEAs). However, the Pareto front (PF) of multiobjective optimization problems (MOPs) affects the performance of MOEAs, especially reference point-based ones. This paper proposes a reference-point-based adaptive method to study the PF of MOPs according to the candidate solutions of the population. In addition, the proportion and angle function presented selects elites during environmental selection. Compared with five state-of-the-art MOEAs, the proposed algorithm shows highly competitive effectiveness on MOPs with six complex characteristics.
\end{abstract}

Keywords: Multiobjective optimization; Many-objective optimization; evolutionary algorithms; genetic algorithms;

\section{1. INTRODUCTION}

Recently, multiobjective evolutionary algorithms (MOEAs), have been proposed to solve multiobjective optimization problems (MOPs) [39]. Generally, MOPs, which involve more one conflicting objective to be optimized, can be formulated as follows:

$$
\min _{x \in D} \underline{F}(x)=\left(\underline{f}_{1}(x), \underline{f}_{2}(x), \ldots, \underline{f}_{M}(x)\right)^{T}
$$

2 where $D \subseteq \mathbb{R}^{n}$ is the decision space; $x=\left(\underline{x}_{1}, \underline{x}_{2}, \ldots, \underline{x}_{n}\right) \in D$ is the decision variable; $M \geq 2$ is the number 3 of the objectives, and $F$ consists of $M$ objectives. The rapid development of MOEAs directly reflects the need to handle MOPs in real-world scenarios, such as in optimizing visualization [2.3, 8, 2.5], traffic control [65] [31], control system design [51] [19], and industrial planning [1, 20, 22, 24]. MOEAs can find a set of

\footnotetext{
${ }^{*}$ Corresponding author: Liuwei Fu

Email address: fuliuwei1@Gmail.com (Liuwei Fu)
} 
optimal solutions (rather than a single one) in an evolutionary manner and have performed excellently on various MOPs.

In the development of MOEAs, Pareto-based approaches, such as dominance relation and density estimation, are first used to optimize MOPs. dominance relation selects a solution by the objectives' values and provides selection pressure toward the Pareto front $(\mathrm{PF})$. Density estimation is used to maintain diversity when some solutions are non-dominated according to the dominance relation.Deb et al.[34] proposed the non-dominated sorting generic algorithm II (NSGA-II) for conflicting problems. The NSGA-II has two main phases: first, a nondominated sorting approach is used to create Pareto rank; then a crowding distance is applied to implement density estimation. Zitzler et al.[16] modified the density estimation and presented an enhanced archive truncation method. However, it is a problem that Pareto dominance-based methods lead to the severe loss of selection pressure on the PF as the number of objectives increases. The primary reason behind the failure of Pareto-based MOEAs is the large increase of non-dominated solutions [53]. Another major reason is that the diversity of a population is hard to maintain with the limited population size in MOPs. Some studies have pointed out the drawback of Pareto-based algorithms [39] [7] [63]. To overcome the drawback of Pareto-dominance-based MOEAs, relaxed-dominance-based approaches have been proposed, such as epsilon dominance [45] [44], favor relation [69] [49], fuzzy Pareto dominance [43] [3], particle swarm[61] [26] [50], and SDE [47].

Indicator-based approaches adopt a performance indicator to optimize a desired ordering among the population set during the evolution. These approaches seem to be a direct way to solve MOPs. Among the current indicators available, the indicator-based EA (IBEA) [14] uses a single indicator to guide the search process. The hypervolume (HV) [13] is probably the most popular performance indicator in multiobjectve search due to its potential to balance convergence and diversity. A number of well-established indicator-based MOEAs that employ the theoretical properties of HV are available, such as the S metric selection evolutionary algorithm (SMS-EMOA) [48] and multiobjective covariance matrix adaptation evolution strategy (MO-CMAES) [10]. Nevertheless, there are two downsides to indicator-based MOEAs in handling MOPs[62]. One is that the computational cost of HV grows exponentially with an increase in the number of objectives [36]. The other is too much selection pressure, which produces inferior performance in terms of distribution on the PF [46] [11]. In such conditions, the HV prefers to choose the knee and border of the PF rather than the points having more diversity [4] [68]. To relieve the computational cost of HV, researchers use the indicator values of estimated HV to improve efficiency. For example, the hypervolume estimation (HypE) algorithm is widely applied in high-dimensional optimization problems by using a Monte Carlo simulation [42]. Recently, methods using several other performance indicators as a substitute have achieved similar results to those using the HV in the evolutionary process. For example, R2 and additive approximation [57] [5].

Decomposition-based approaches divide complex MOPs into a set of single-objective sub-problems through aggregation functions, and then solve them simultaneously in a collaborative manner. The evolution direc- 
tion of a sub-problem is guided by specifying a set of well-distributed reference points that maintain the population' diversity [59] [6] [66]. Decomposition-based approaches can be directly applied to the selection mechanism of MOEAs by a single scalar value rather than pareto dominance. Generally, weight vectors and the aggregation function are critical to decomposition-based approaches [40] [67] [38]. Weight vectors affect the distribution of the solutions in the objective space, and guide the evolutionary direction. The aggregation function provides the mechanism to update individuals in the selection strategy. Recently, two types of decomposition-based approaches were classified [64]. The first type divides MOPs into a set of singleobjective problems (SOPs). For example, Multiple Single Objective Pareto Sampling(MSOPS) and MOEA based on decomposition (MOEA/D) [52],NSGA-III [33]. The second type of decomposition-based approach divides MOPs into a set of sub-MOPs. For example, a multiobjective evolutionary algorithm using dynamic weight design method (MOEA/D-M2M) [17]. Although decomposition approaches have become popular due to their efficiency [47], they encounter difficulty in handing an irregular PF [7].

Although many decomposition approaches based on adaptation have been proposed to solve MOPs with irregular PFs, it is hard to maintain a balance between convergence and diversity. Motivated by the ideas, we propose a new adaptation reference-point-based optimization algorithm, (ARMA), for solving MOPs. Compared with existing reference-point-based methods, the main contributions of this paper are as follows:

- A hyperplane learning strategy is proposed to adjust the relative position of reference points to deal with MOPs that have a concave or convex PF. The proposed strategy uses a parameter $\phi$ to control the hyperplane shape of the reference points according to the objective value of a whole population so that the distribution of solutions can be improved.

- The new clustering method is designed to balance the niche-preservation operation in multiobjective optimization according to the reference points. We divide a population into a number of niches by value of the proportion and angle, and then select a better solution in the niche. In this method, convergence and diversity are primarily measured by proportion and angles.

The remainder of this paper is organized as follows. Section II reviews related work. The details of the proposed ARMA are described in Section III. Section IV present results and an investigation of multiobjective problems. Further discussion of parameter $\phi$ behavior is given in Section V. Finally, conclusions are drawn in Section VI.

\section{RELATED WORK}

Reference point-based MOEAs have been proposed in which the quality of the population is measured by a set of reference points. Two phases (constructing a reference set and measuring the quality of the population) are the main methods used in reference-point-based approaches. In NSGA-III [33] [29], the reference points, 


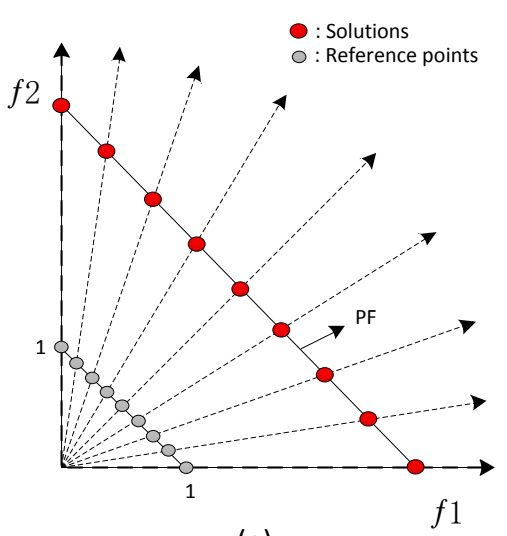

(a)

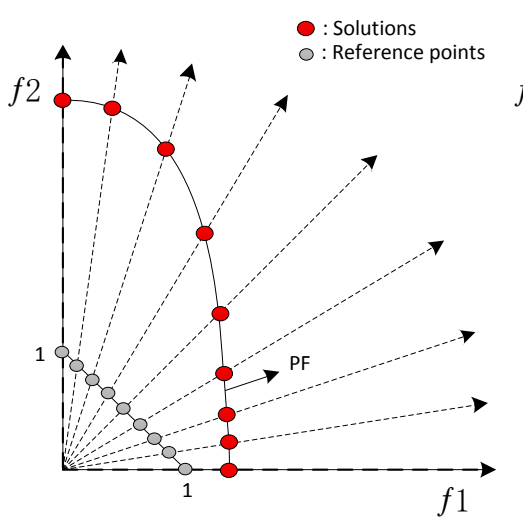

(b)

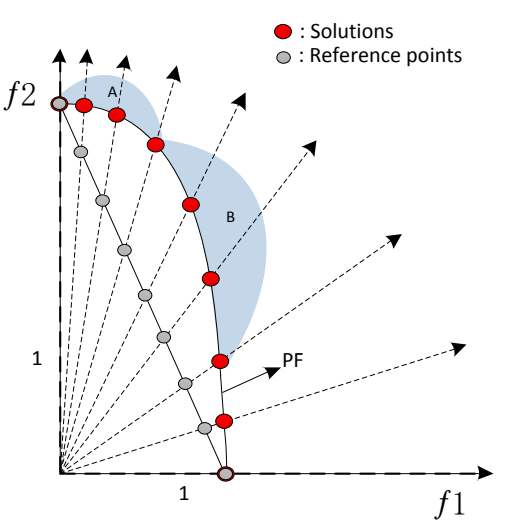

(c)

Figure 1: Pareto-optimal solutions (red solid dots) specified by different reference points (grey solid dots). (a) Pareto-optimal solutions specified by nine uniformly distributed reference points on a PF with the same range.(b) Pareto-optimal solutions specified by nine uniformly distributed reference points with differently scaled.(c) Pareto-optimal solutions specified by nine adapted reference points in the RVEA algorithm.

using Das and Dennis's systematic approach [27], are predefined to ensure the population's diversity. NSGAIII's selection strategy uses the Pareto dominance relation and decomposition operator to balance convergence and diversity in the evolutionary process. In RVEA [54], a scalarization approach using angles and distances is adopted to measure solutions. In a strength pareto MOEA based on reference direction (SPEA/R) [58], a reference vector-based local fitness assignment scheme preserves the most promising individual in the subregion. Tian et al.[60] adapted the position of the reference point to preserve extreme solutions for better diversity. In indicator-based MOEA (AR-MOEA), an excellent individual from the archive is used to replace the poorly distributed reference point and serves as the reference point to guide the evolutionary search.

Reference-point-based MOEAs maintain the distribution of the population through a clustering strategy using reference points as shown in Fig. 1(a). In general, the diversity of predefined reference points is crucial to the PF. Das and Dennis's systematic approach, which places points on a $f_{1}+f_{2}+\cdots+f_{M}=1$ hyperplane, is adopted in most MOEAs to generate the predefined reference points[9] [56]. However, in Fig . 1(b), it is difficult to generate uniform reference points on differently scaled PF of MOPs, as is the case in the WFG test problems [55] and the scaled DTLZ problems [35] which have various features. Different scale optimization problems cause great difficulties for reference-point-based selection strategies.

There are two methods to address this issue. One is that objective normalization dynamically is introduced as the search proceeds, as in NSGA-III, SPEA/R and $\theta$-DEA. The other is to adapt the reference points according to the ranges of the objective values. In RVEA, reference point adaptation is used to deal with the badly-scaled PF in Fig. 1(c). These two methods have obtained better performance on the scale problem. 
However, these methods do not achieve a relatively uniform distribution of solutions when coping with a non-planar PF, especially concave and convex PFs. From Fig. 1(c), it can be seen that the individual distributions of areas A and B perform differently. Compared with the A area, solutions in the B area are too sparse. Maintaining the diversity in the peak of the PF is becoming a new challenge for reference point-based MOEAs.

\section{PROPOSED ALGORITHM}

The basic framework of the proposed ARMA is described in Algorithm 1. At the beginning of ARMA, the population is initialized randomly and the reference points are constructed using Das and Denis's systematic approach and Two-layer methods. In each iteration, the parent population reproduces its offspring by crossover operation and mutation operation. Then, the parent and offspring are integrated into the double population. Next, in order to handle problems with disparately scaled objectives, the adjustment strategy dynamically adjusts the distribution of reference points according to the obtained solution on the approximated PF. Then the solution in the double population applies proportion [41] and angle to link up with one reference point. The double population is partitioned into $N$ different subpopulations, where $N$ is the number of the population. The proportion and angle are described in subsection B. After the clustering operation, the candidate solution with the smallest Fitness values is chosen in the environmental selection. In the following sections, the important procedures of the ARMA are described in detail.

\subsection{Adaptive Methods}

We are proposing new adaptation methods which comprise two steps to maintain the diversity of reference points. First, the reference points are adjusted for the scale problems and form the approximate shape of the $\mathrm{PF}$ by using the parameter $\phi$. Further performance of parameter $\phi$ is described in Section V. Second, the relative position of the reference points must be reevaluated by its neighbor.

In the early period, the maximum values of each objective function is obtained to deal with badly-scaled $\mathrm{PF}$ in RVEA, but the worst individual in the population severely affects this implementation and fluctuates strongly in the maximum values. Thus, we use small steps to readjust the reference point. The detailed introduction of small step $S$ and reference point adjustment are in equation (2).

$$
S=\operatorname{argmin}\left(Z_{j}^{\max }-Z_{j-1}^{\max }\right), 1<j \leq M,
$$

where $Z_{j}^{\max }$ denotes the maximum values of $j$ th objective function; $M$ is the number of objectives; and $S$ is the increment in the objective. Therefore, the maximum value of each objective function is readjusted as follows:

$$
Z_{j}^{\max ^{\prime}}=Z_{1}^{\max }+S *(j-1), 1<j \leq M,
$$




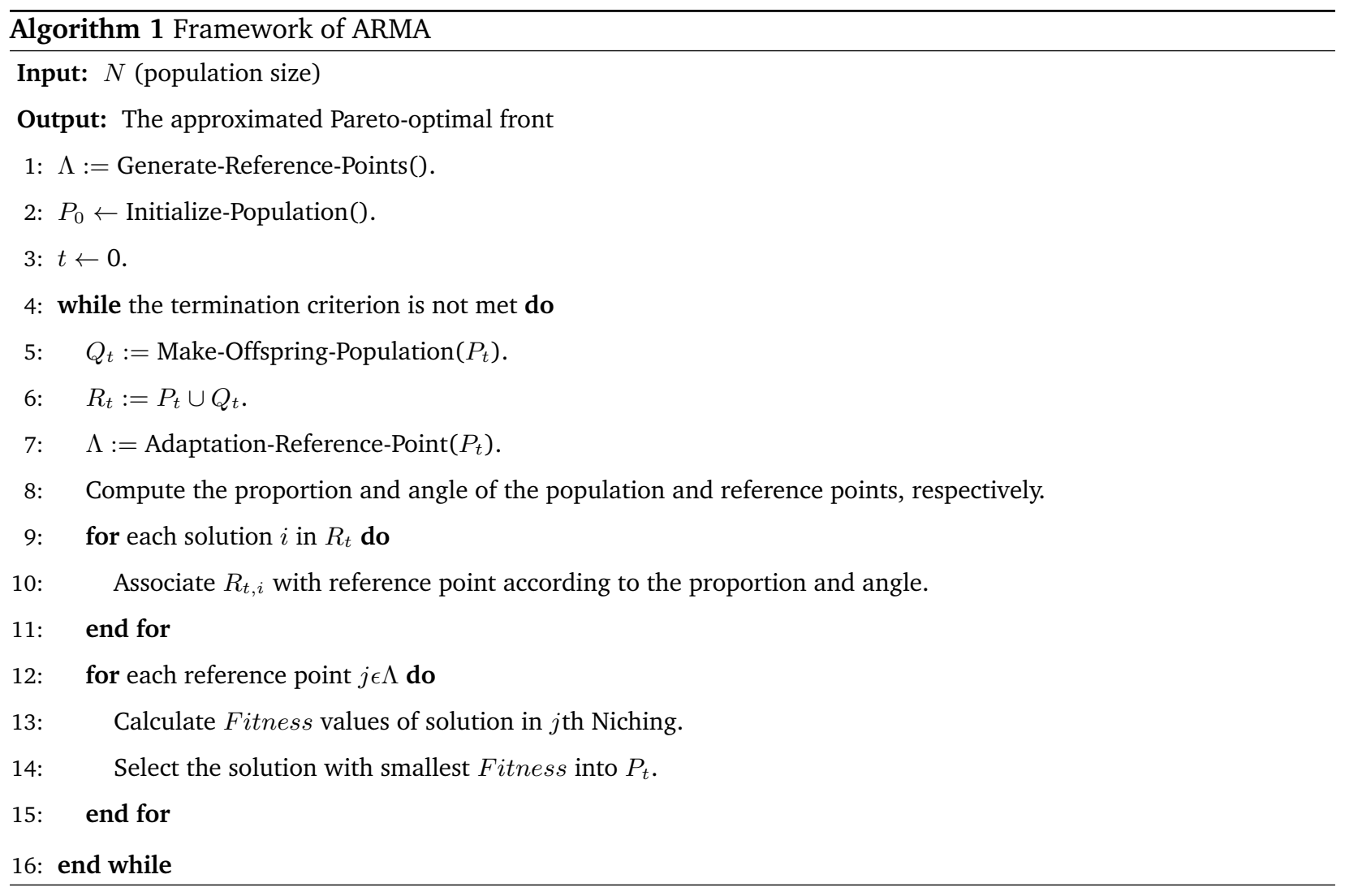

where modified $Z_{j}^{\text {max }}$ is the maximum value of $j$ th objective function. After that, the relative position of the reference point is adjusted by the vector $Z_{t}=\left(Z_{1}^{\max ^{\prime}}, Z_{2}^{\max }, \ldots, Z_{m}^{\max }\right)$ for the first time in the following manner:

$$
R_{t, i}=R_{0, i} \circ Z_{t}, 1<i \leq N
$$

where $R_{t, i}$ denotes the $i$ th adapted reference point in the $t$ generation ; $R_{0, i}$ denotes the $i$ th reference point generated in the initialization stage; $N$ is the number of the reference points, and the ' $o$ ' operator denotes the Hadamard product, which wisely multiplies two matrices of the same size. In order to make the reference point satisfy the situation that the surface of the PF is curved, as shown in Fig. 2(a), the reference point moves $\phi$ length of the distance between the reference point and the nearest individual in its niche along the direction of the origin and the current reference point.

$$
R_{t, i}=R_{t, i}\left(\frac{\left\|R_{t, i}\right\|+\phi D}{\left\|R_{t, i}\right\|}\right),
$$

where $D$ is the distance between the reference point $R_{t, i}$ and the individual in $R_{t, i}$ niche. The hyperplane that the modified reference points make up is pretty similar to the shape of the PF. However, the distribution of the reference points on the hyperplane is not as evenly even as before the modification process in Eq (5). The second modification process is therefore required to make the reference points distribute evenly on the 


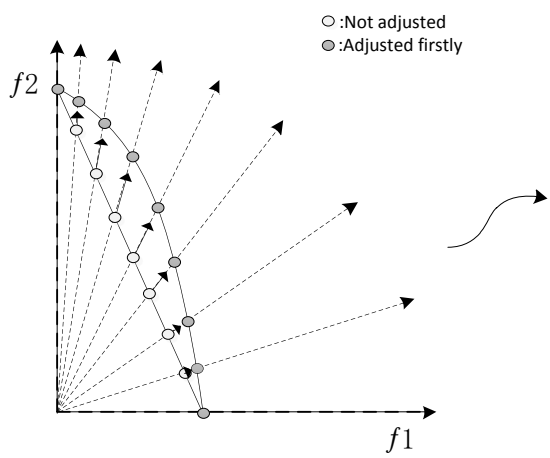

(a)

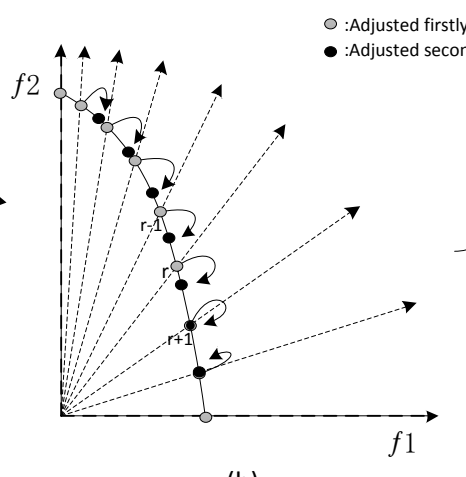

(b)

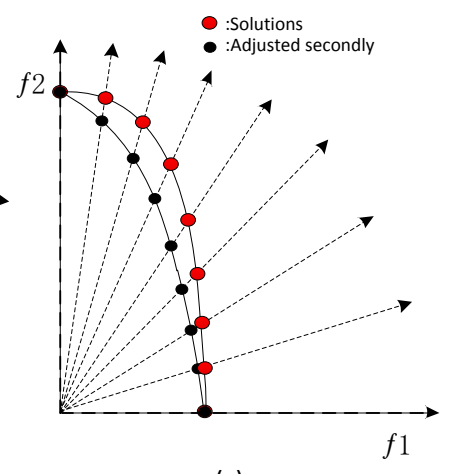

(c)

Figure 2: Adaptation reference points according to the solutions obtained. (a) nine reference points move to PF. (b) adjusting the reference points according to their $m$ neighbor. (c) Pareto-optimal solutions specified by nine adapted reference points.

hyperplane. The detailed adjustment process of the reference points is as follows. First, for each reference point, the $m$ neighbor is selected with maximum distance from the $2 m$ neighbor, where the $2 m$ nearest neighbor reference points along positive and negative directions of the coordinate axis are found. Then, the average values of all points' Euclidean distances from the points to their $m$ neighbors in $m$ directions are calculated. If there are not any reference points in the positive direction for some reference points, take the boundary points for example, a value of average is assigned. Lastly, two reference points $A$ and $B$ with the largest distance in the $m$ th objective are selected. In order to make their distance reach the average, $A$ is adjusted to move $B$, and $A$ and $B$ are marked, where $B$ must meet the following conditions:

- condition 1: $B$ has a value of 0 in the $m$ th objective (boundary point).

- condition 2: $B$ is marked.

- condition 3: if conditions 1 and 2 are not satisfied, $B$ is randomly selected from $A$ and $B$.

This way, each reference point has the same approximate distance from its neighbors, thus having uniform distribution. The detailed procedure is introduced in Algorithm 2.

It is worth emphasizing that the proposed adaptation method is different from other reference-pointbased evolutionary algorithms, such as indicator-based MOEA (AR-MOEA). Compared with AR-MOEA, the proposed adaptation method adjusts reference points to form an approximated PF in this paper. In addition, the relative positions of the reference points are reevaluated by their neighbors. However, the goal of ARMOEA is to preserve the extreme solutions for better diversity in the adjusting approach. 


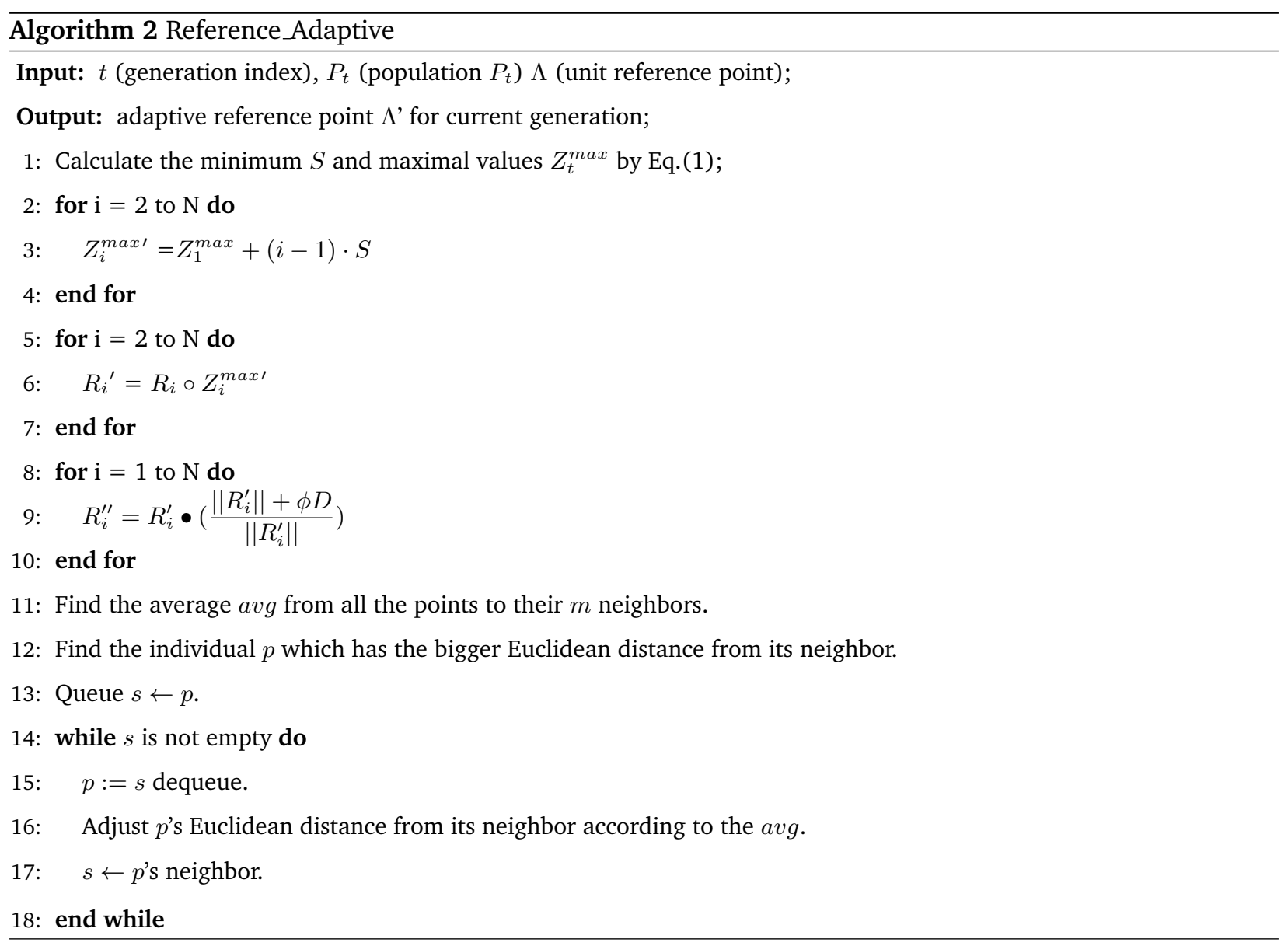



calculated as

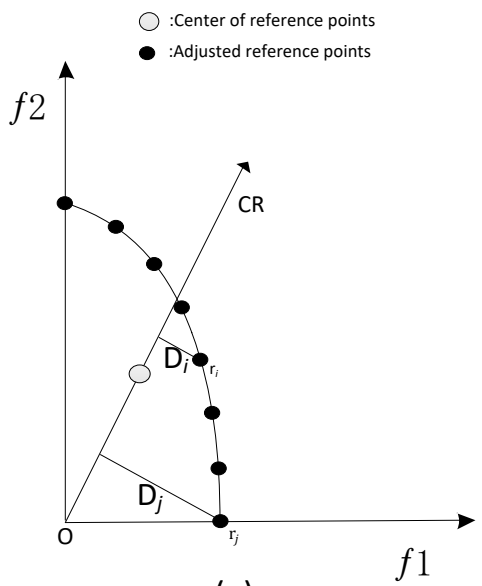

(a)

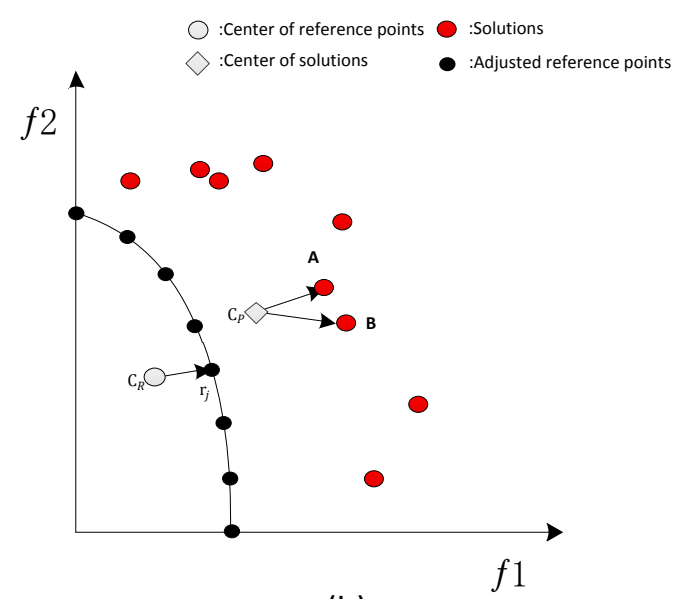

(b)

Figure 3: Example showing the calculation of the proportion and angle.

$$
P R_{i}=\frac{D_{i}}{\max \left(D_{1}, D_{2}, \ldots, D_{2 N}\right)},
$$

\subsection{Selection Strategy}

There are many ways to divide the entire population into $N$ subpopulations according to the reference points. In NSGA-III, the perpendicular distance between each solution and reference point is presented. In RVEA, the angle between the solution and reference point is used to complete the clustering operation. Compared with the aforementioned approaches, the ARMA partitions the population into $N$ subpopulations using the approximation of proportion and angles, and the selection strategy is implemented separately in each subpopulation. The proportion and angles method can effectively avoid the impact of non-normalization and form a mapping relationship between reference points and the individual to complete the partition in emphasizes the center of the population. In the proposed ARMA, the environment environmental selection operation comprises two steps: calculating the proportion and angle, and selecting an elite individual inside the subpopulations.

\subsubsection{Calculating the proportion and angle}

The center of population, or reference sets, denoted as $C_{P}$ or $C_{R}$, could be estimated by the objective value of each individual or reference point. The proportion of $i$-th reference point, denoted as $P R_{i}$, can be 
as $P S$, can be calculated using the same methods.

The angle between vector $\overrightarrow{r_{i}-C R}$ and vector $\overrightarrow{f-C P}$ can distinguish the relationship whether they have the same direction or not. $\mathbf{f}$ is an individual in the population. The $\overrightarrow{r_{i}-C R}$ and the $\overrightarrow{f-C P}$ can be considered as the same direction vector if they have the smallest angle. Given two vectors, a large cosine value means a smaller angle. The cosine value can be calculated as

$$
\cos \theta_{i, j}=\frac{\overrightarrow{f_{j}-C P} \cdot \overrightarrow{r_{i}-C R}}{\left\|f_{j}-C P\right\| \cdot\left\|r_{i}-C R\right\|},
$$

where $\theta_{i, j}$ is the angle between the $\overrightarrow{r_{i}-C R}$ and the $\overrightarrow{f-C P}$.

\subsubsection{Selecting an elite individual inside the niche}

Population $P_{t}$ is divided into $N$ niches according to the proportion value and angle. Individual $f$ is allocated to a reference point $r$ if the angle and difference of proportion $\left|P S_{f}-P R_{r}\right|$ between individual $f$ and reference point $r$ is minimal. $P S_{f}$ and $P R_{r}$ is the proportion of $f$ and $r$, respectively. In other words, individual $f$ and reference point $r$, which have the smallest angle and proportion difference, are linked together.

After partitioning the population, it becomes important to select individuals from the subpopulation. Most current algorithms based on reference vectors use the distance or angle between the individual and the reference vector to select candidate individuals. In this paper, we propose an approach as

$$
\text { Fitness }_{f, r}=\text { Distance }_{f, r}+\text { Angle }_{f, r}+\left|P S_{f}-P R_{r}\right|,
$$

where Distance (Angle) is the Euclidean distance (angle) between the individual and reference point in the niche, respectively. $P S_{f}$ and $P R_{r}$ are the proportion of individual $f$ and reference point $r$.

The Fitness function is designed to meet the convergence and diversity criteria. In the early stage of the Fitness function, providing strong convergence pressure makes the population quickly converge to the PF during the evolutionary process. Distance $_{f, r}$ plays a decisive role in the convergence so that the whole population can converge to the PF quickly. At a later stage, the mechanism of Angle $e_{, r}+\left|P S_{f}-P R_{r}\right|$ becomes more crucial than the convergence and provides a uniformly distributed solution set for decision makers. This means that the diversity criterion strongly affects the value of Fitness and plays a decisive role. In addition, although the solution sets are not evenly distributed in the early stage, the population is divided into $N$ niches in the early evolutionary process, which roughly guaranteed the distribution. The balance of convergence and diversity can be maintained by selecting the elite individuals based on the smaller Fitness value in each niche.

\subsection{Computational complexity of the ARMA}

In order to analyze the computational complexity of the ARMA, we only consider the main steps and processes in one generation in Algorithm 1. The computational complexity of ARMA is composed of two 


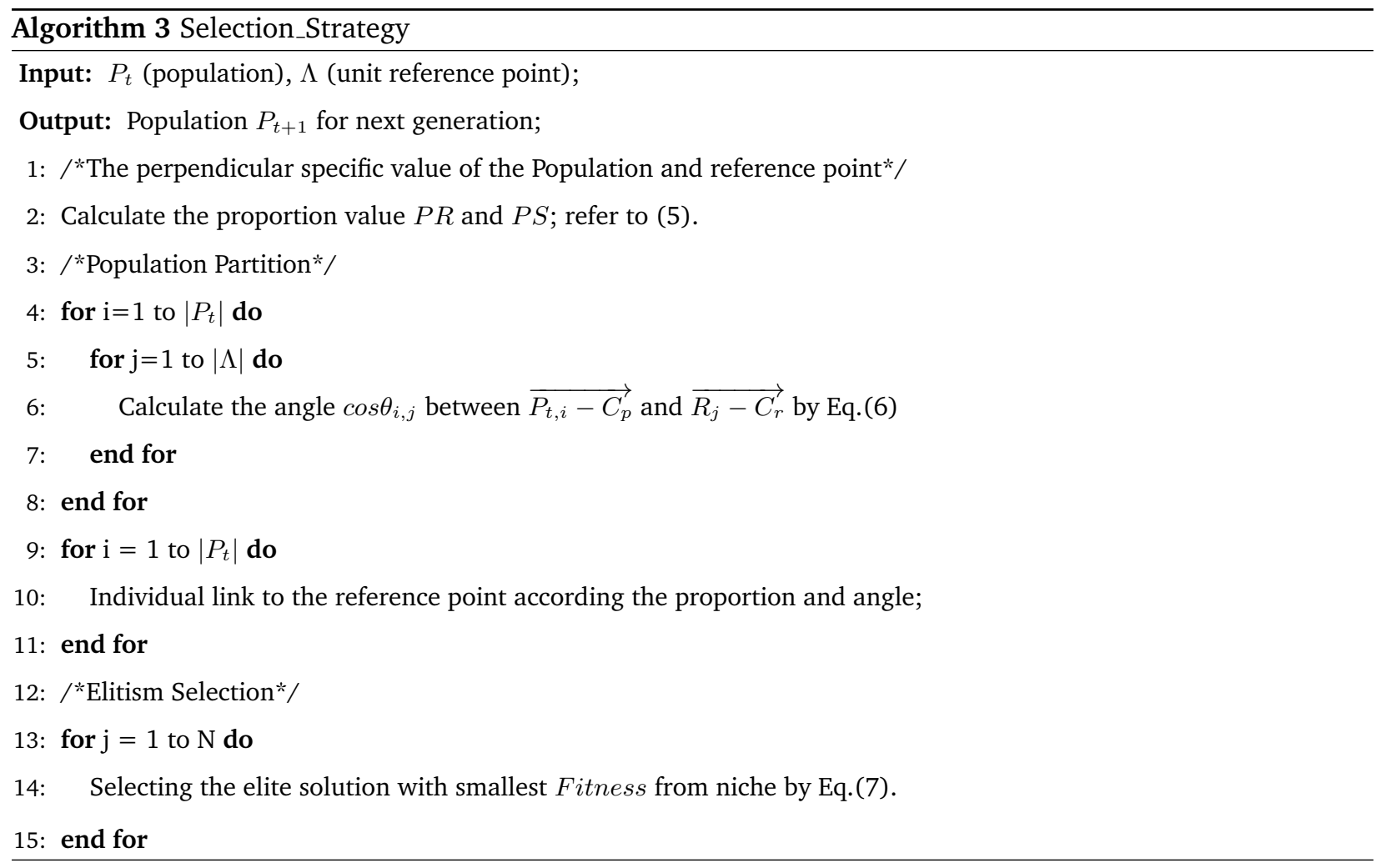

parts: reference-point adaptation and environmental selection.

In Algorithm 2, the computational complexity of the reference points' adaptation is $O\left(M N^{2}\right)$ when all the reference points need to be adjusted; $N$ is the number of solutions, and $M$ is the number of the problem's objectives. In addition, environmental selection consists of partitioning the population and the elite selection. In population partition, the proportion and angle calculations take $O(2 M N)$ and $O\left(M N^{2}\right)$ in the worst case, respectively. Finally, the calculation of Fitness and elite selection just spends $O(2 N)$ in a niche in the worst scenario.

Therefore, the worst case average computational complexity of ARMA within one generation is $O\left(M N^{2}\right)$, which is as efficient as RVEA and NSGA-III.

\section{Comparative Studies}

\subsection{Performance Metrics}

In order to test the performance of the algorithm in the experiment, we used the well-known indicators IGD [21] and HV [13]. The details of IGD and HV are introduced next. 


\subsubsection{Inverted Generational Distance}

Inverted generational distance (IGD) can test the population obtained by the MOEA convergence information and distribution information simultaneously. Let $A$ be a set of solutions uniformly sampled from the true $\mathrm{PF}$, and $P_{*}$ be the approximated solutions in the objective space. The IGD calculated as follows:

$$
\operatorname{IGD}\left(A, P^{*}\right)=\frac{1}{\left|P^{*}\right|} \sqrt{\sum_{j=1}^{\left|p^{*}\right|} \widetilde{d}_{i}^{2}}
$$

where $d_{i}\left(\widetilde{d}_{i}\right)$ is the Euclidean distance between the $i$-th member in set $A$ and its nearest member in set $P_{*}$.

\subsubsection{Hypervolume}

The HV metric is used to measure the volume of the objective space. In general, HV can measure both the convergence and diversity of a solution set in the last generation. Let $P^{*}$ be a set of approximated solutions in the objective space, and $r=\left(r_{1}, r_{2}, \ldots, r_{m}\right)_{T}$ be a reference point which is dominated by all individuals in set $P^{*}$. The HV is calculated as follows:

$$
H V\left(P^{*}, r\right)=\text { volume }\left(\bigcup_{f \in P^{*}} f\left[f_{1}, r_{1}\right] \times \ldots\left[f_{m}, r_{m}\right]\right),
$$

In addition, we calculated HV exactly using the recently proposed WFG algorithm [42] for problems with no more than 8 objectives. The Monte Carlo simulation proposed in [32] was adopted to approximate the HV for problems having 12 objectives, and 10,000,000 sampling points were used to ensure accuracy.

\subsection{Experimental Settings}

\subsubsection{Reproduction Parameters}

For the comparison in the experiment, all simulated binary crossover and polynomial variations in the algorithms' settings were the same. The crossover probability and its distribution index were $p_{c}=1.0$ and $\eta_{c}=20$. Similarly, the polynomial mutation probability and distribution were set to $p_{m}=1 / n$ and $\eta_{m}=20$, respectively.

\subsubsection{Population Size}

The population size in 2-, 3-, 5-, 8- and 12-objectives was set to 100, 105, 126, 120, 110, respectively.

\subsubsection{Termination Condition}

The termination condition is set according to the maximum generation. The maximum number of generations was set to 500 for ZDT suites problem, and for the DTLZ and WFG suites problems, the maximum generation was 1000. In addition, each algorithm ran independently 30 times for each problem. 
4.3. Performance on Multiobjective Optimization problems

4.3.1. Test Problems and Other Algorithms in Comparison

In order to study the performance of the ARMA algorithm on multiobjective problems, the test problems ZDT1, ZDT3 and ZDT6 were selected from the ZDT suites [15]. The ZDT multiobjective problems contain two objectives and all scaled decision variables. They were used to test the ability of MOEAs on the two objectives. ZDT1 has a convex PF with different partial constraints. ZDT3 is a non-continuous, convex PF test problem and poses a great challenge to the MOEAs.

The ARMA algorithm is a reference-point-based decomposition algorithm instead of being referencevector-based, so we chose MOEA/DD [37] and MOEA/D as contrasting algorithms for the multiobjective problems. MOEA/D is representative of the decomposition algorithm, and it has advantages in multiobjective algorithms. MOEA/DD exploits the merits of both dominance- and decomposition-based approaches to balance the convergence and diversity of the evolutionary process.

Table 1: MEAN AND STANDARD DEVIATION IGD AND HV VALUES ON ZDT PROBLEMS

\begin{tabular}{|c|c|c|c|c|c|c|}
\hline \multirow{2}{*}{ Prob. } & \multicolumn{3}{|c|}{ IGD } & \multicolumn{3}{|c|}{$\mathrm{HV}$} \\
\hline & ARMA & MOEA/D-PBI & MOEA/DD & ARMA & MOEA/D-PBI & MOEA/DD \\
\hline ZDT1 & $3.6444 \mathrm{E}-3(0.000022)$ & $4.3361 \mathrm{E}-3(0.009302) \ddagger$ & $3.6608 \mathrm{E}-3(0.000586) \ddagger$ & $3.6616 \mathrm{E}+0(0.000253)$ & $3.6540 \mathrm{E}+0(0.043238) \ddagger$ & $3.6579 \mathrm{E}+0(0.010103) \ddagger$ \\
\hline ZDT2 & $3.6558 \mathrm{E}-3(0.000004)$ & $4.4443 \mathrm{E}-3(0.027939) \ddagger$ & $3.6653 \mathrm{E}-3(0.080872) \ddagger$ & $3.3291 \mathrm{E}+0(0.060728)$ & $3.3248 \mathrm{E}+0(0.17208) \ddagger$ & $3.3286 \mathrm{E}+0(0.063756) \ddagger$ \\
\hline ZDT3 & $1.4661 \mathrm{E}-1(0.000126)$ & $1.9661 \mathrm{E}-2(0.011933)$ & $6.8035 \mathrm{E}-2(0.01182)$ & $3.7964 \mathrm{E}+0(0.000654)$ & $4.6792 \mathrm{E}+0(0.057257)$ & $4.6560 \mathrm{E}+0(0.069763)$ \\
\hline ZDT4 & $3.8800 \mathrm{E}-3(0.000194)$ & 4.5407E-3(0.001969)‡ & $3.9164 \mathrm{E}-3(0.007304) \ddagger$ & $3.6609 \mathrm{E}+0(0.001205)$ & $3.6482 \mathrm{E}+0(0.006511) \ddagger$ & $3.6552 \mathrm{E}+0(0.312785) \ddagger$ \\
\hline ZDT6 & $4.2466 \mathrm{E}-2(0.000014)$ & $4.5755 \mathrm{E}-2(0.001211) \ddagger$ & $4.2525 \mathrm{E}-2(0.035154) \ddagger$ & $3.0400 \mathrm{E}+0(0.159858)$ & $3.0327 \mathrm{E}+0(0.006087) \ddagger$ & $3.0408 \mathrm{E}+0(0.00293)$ \\
\hline
\end{tabular}

$\ddagger$ and $\dagger$ indicate ARMA performs significantly better than and equivalently to the corresponding algorithm, respectively.

\subsubsection{Results on Multiobjective Optimization Problems}

The mean and standard deviation values of IGD and HV of the current population were used to evaluate the ARMA, MOEA/DD, and MOEA/D-PBI, where the best value for each problem is marked in grey background. In addition, in order to accurately analyze the statistical results, the Wilcoxon rank-sum test was carried out to indicate the significance between different results at the 0.05 significance level in multiple comparisons [18].

In the ZDT test suite, ZDT3 is the only disconnected problem. Table I shows that the ARMA performs, in terms of the number of the function evaluations, significantly better than MOEA/DD and MOEA/D-PBI on most of the test problems. Compared with MOEA/DD, the ARMA is better according to HV value with the exception of the ZDT3 and ZDT6 problems. In addition, the ARMA competes well with MOEA/D-PBI and MOEA/DD on problem ZDT3. It is due to the distribution of reference vectors in the ARMA that increases the probability of selecting solutions in sparse regions.

In Fig. 4, approximated solutions over 30 independent runs are given with an intuitive understanding of the performance of the algorithm. All three algorithms could approximate the PF for three problems, but they 

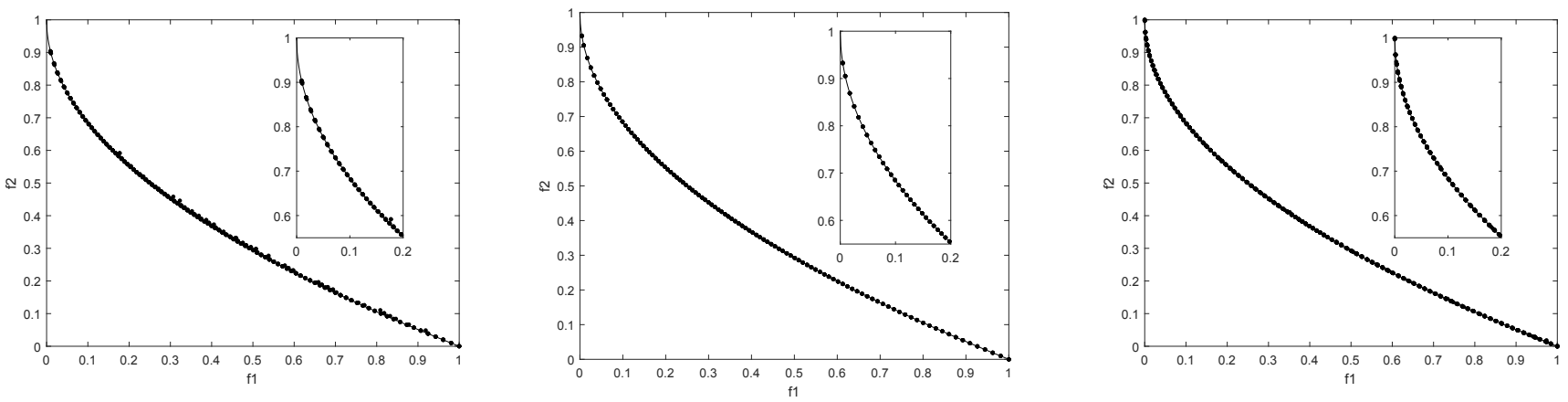

\section{ZDT1}
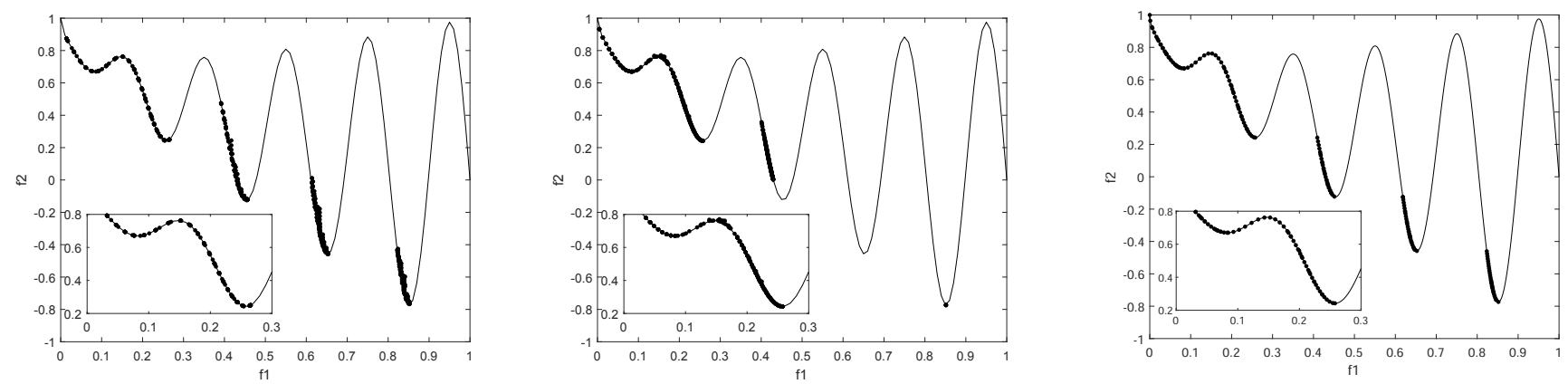

\section{ZDT3}
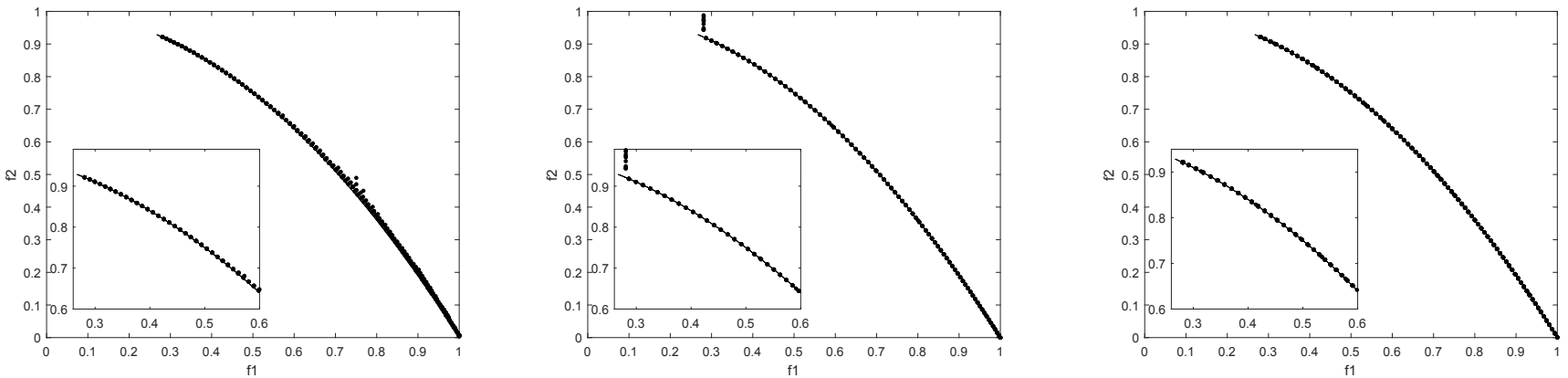

ZDT6

(a) MOEA/D-PBI

(b) MOEA/DD

(c) ARMA

Figure 4: Approximated solutions for ZDT test problems, Left column:MOEA/D, middle column:MOEA/DD; and right column:ARMA

performed differently in terms of convergence and diversity. First, for convergence analysis, the ARMA could approximate all the PF for the ZDT problems, which is significantly better than MOEA/DD and MOEA/DPBI. MOEA/DD could approximate the PF at ZDT1 and ZDT3, but the MOEA/DD produces many dominant resistance solutions on the ZDT6 problem. MOEA/D-PBI had some non-convergence solutions although it 

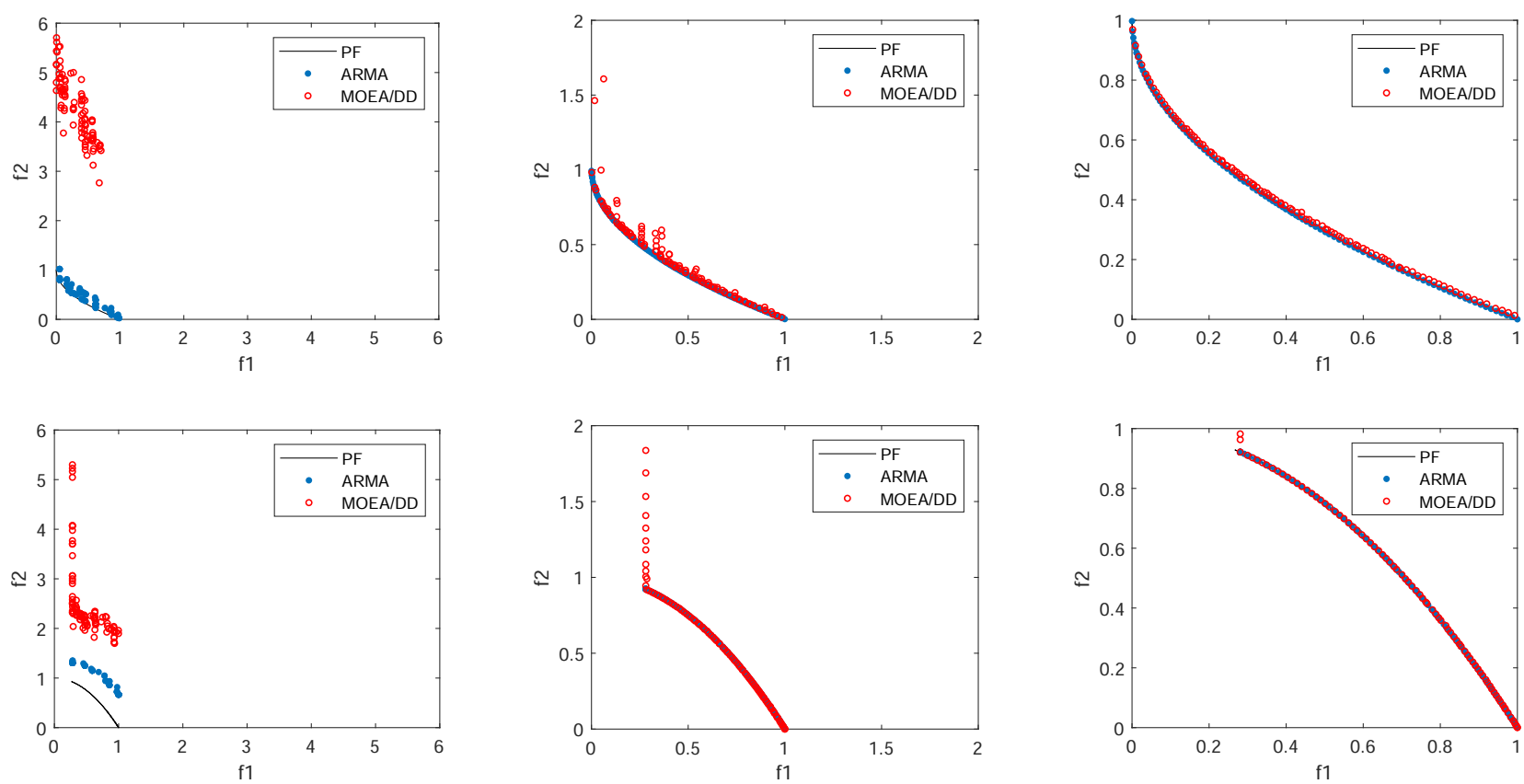

Figure 5: Evolution behavior comparison between ARMA and MOEA/DD for three stages on ZDT4 and ZDT6. Left: 50th generation; middle: 200th generation; and right: 300th generation

could approximate to the PF. Secondly, for the diversity analysis, it can be clearly seen from Fig. 4 that the ARMA was better than MOEA/D-PBI and MOEA/DD on the test problems with sharp shapes. This is mainly due to the adaptation strategy of the ARMA on the PF.

\subsubsection{Comparison of Evolution Behavior With MOEA/DD}

Previous experiments have shown that the performance of the ARMA is more competitive among three algorithms, but the differences are not easy to observe even though they have the same reference-vector (point)-based framework. In order to verify whether there are differences in the evolutionary process, we represented the 50-generation, 200-generation, and 300-generation approximate PF, which were selected by the ARMA and MOEA/DD in the ZDT4 and ZDT6 test problems in Fig. 5. As can be seen from the figure, the convergence and the distribution of the ARMA were significantly better than MOEA/DD in the evolutionary process. This shows that the proportions and distances used in the PPM function of the ARMA to select elite individuals has obvious advantages.

The experiment on the ZDT test suite shows that the ARMA performs significantly better than MOEA/DD and MOEA/D-PBI on most of the test problems. The ARMA exhibits strong competitiveness in multiobjective problems, especially convex functions. 

and 12 .

\subsection{Performance on Many-objective Optimization problems}

In order to illustrate the performance of the ARMA on many-objective problems, a comparison of the ARMA with five state-of-the-art algorithms is given.

\subsubsection{Test Problems}

As a basis for the comparisons, the experimental problems are the two well-known many-objective suites, Deb-Thiele-Laumanns-Zitzler (DTLZ) [35] and Walking Fish Group (WFG) [55]. These test problems have various features, such as having a linear, multi-modal, concave, discontinuous, or degenerate PF [46]. In Table II, we give a detailed classification of the features of the many-objective test problems. In addition, we use the convex function CDTLZ2 [11], which is a modification of the DTLZ2 problem in the experiment. For DTLZ1-6 and CDTLZ2 problems, the number of decision variables was set to $\mathrm{n}=\mathrm{m}+\mathrm{k}-1$. In particular, DTLZ1 was set to $\mathrm{k}=5$, for DTLZ2-6 and CDTLZ2, to $\mathrm{k}=10$. In addition, the number of objectives was 2,3,5,8,10

Table 2: FEATURES OF THE TEST PROBLEMS

\begin{tabular}{|c|c|}
\hline Problem & Features \\
\hline DTLZ1 & Linear, Multi-modal \\
\hline DTLZ2 & Concave \\
\hline DTLZ3 & Concave, Multi-modal \\
\hline DTLZ4 & Concave, Degenerate \\
\hline DTLZ5 & Concave, Degenerate, Biased \\
\hline DTLZ6 & Mixed, Biased \\
\hline CDTLZ2 & Concave, Multi-modal \\
\hline WFG1 & Concave, Deceptive \\
\hline WFG2 & Convex, Discontinuous, Nonseparable \\
\hline WFG3 & Linear, Degenerate, Nonseparable \\
\hline WFG4 & Concave, Nonseparable \\
\hline WFG5 & Concave, Biased \\
\hline WFG6 & Concave, Noseparable, Biased \\
\hline WFG7 & \\
\hline WFG8 & \\
\hline WFG9 & Concave, Noseparable, Deceptive, Biased \\
\hline
\end{tabular}




\subsubsection{Other Algorithms in Comparison}

In the comparison experiment of the ARMA, we chose the latest, promising MOEAs as a basis comparison. Therefore, five algorithms, RVEA [54], SPEA2+SDE [47], NSGA-III [33], MOEA/D [52], and HypE [32], represent different types of heuristics, respectively. The five state-of-the-art algorithms were considered to be the peer algorithms, and a brief description of each algorithm is given next.

- RVEA [54]: The angle-penalized distance (APD) function is formed by the angle and the distance to balance the population convergence and diversity in high-dimensional space. In addition, reference vectors were dynamically adjusted by using the maximum value of the population in each objective, so it could cope with the scale of the multiobjective problem.

- SPEA2+SDE [47]: SDE is a well known dominance-based algorithm. It uses a density-based estimation method that considers both the convergence and distribution information of individuals to provide strong selection pressure in high-dimensional space. SPEA2+SDE also have good performance of convergence and distribution under the conditions when the MOP's PF is irregular.

- NSGA-III [33] : NSGA-III is an improved version of NSGA-II. NSGA-III can not only solve non-dominant disadvantage in high-dimensional space, but also greatly improves the distribution of the population in the algorithm. The NSGA-III uses a reference vector to generate niches so as to associate and select elite individuals. This is the main reason for comparison to the ARMA test.

- MOEA/D [52]: The MOEA/D algorithm is the representation of decomposition-based algorithms and has greater competitiveness in MOEAs. The penalty-based boundary intersection (PBI) is the most promising aggregate function because it can provide a balance between convergence and the distribution maintenance mechanism during the process of environmental selection.

- HypE [32]: HypE is a new hypervolume-based evolutionary algorithm for many-objective optimization, which uses HV indicators as criteria to choose elite individuals during environmental selection. In HypE, the non-dominated solutions are compared according to their hypervolume-based fitness values.

\subsubsection{Experimental Results and Analysis}

The HV results (mean and standard) are given in Table III for the six algorithms on all six MOPs categories, convex, concave, linear, discontinuous, degenerate, and mixed, respectively. The better mean is highlighted in grey background on each test instance. A detailed analysis is shown for each category for a number of test problems.

\section{- Convex Pareto Front}

We chose CDTLZ2 as a test MOP for the convex. As can be seen in Table III, the ARMA algorithm shows a clear superiority over the other algorithms on these problems. 
Table 3: MEAN AND STANDARD DEVIATION HV VALUES OBTAINED BY SIX ALGORITHMS FOR DTLZ and WFG PROBLEMS ON 2, 3, 5, 8 AND 12 OBJECTIVES.

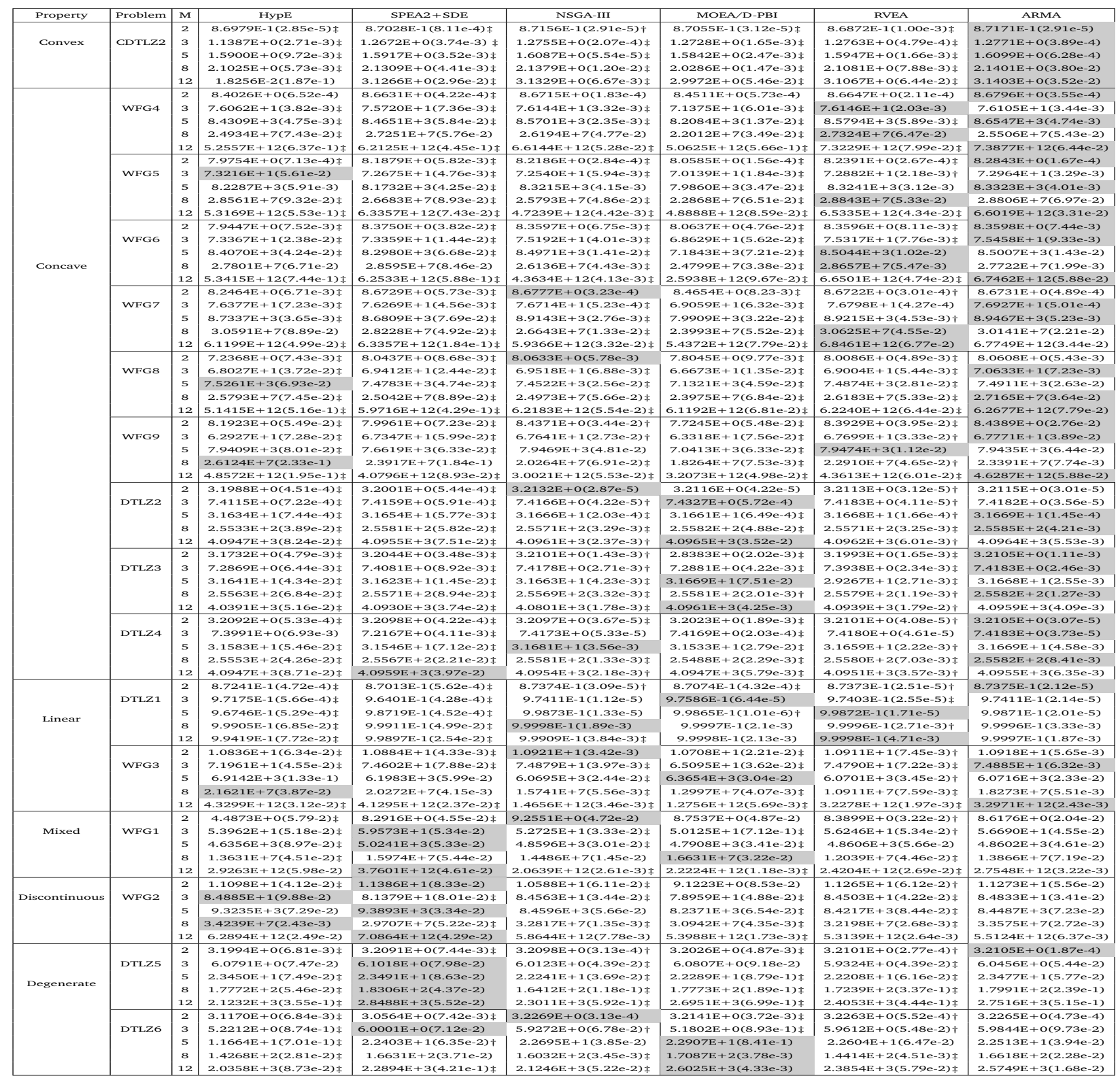

$\ddagger$ and $\dagger$ indicate ARMA performs significantly better than and equivalently to the corresponding algorithm, respectively.

The 3-dimensional figure is given for the diversity of convex problem CDTLZ2. We can see that the ARMA algorithm achieved the best performance on the CDTLZ2 instances from Fig. 6. The ARMA had the best solution in the boundary of the PF. However, MOEA/D-PBI, SPEA2+SDE, NSGA-III, and RVEA had very poor performance in this situation, although their overall distribution was better than HypE. To describe the distribution of obtained solutions in the high-dimensional objective space, we used 12- 


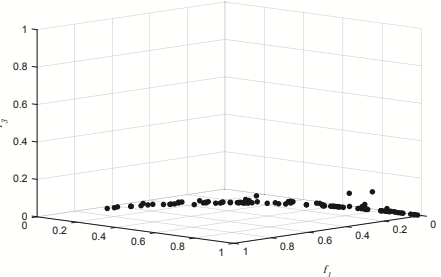

HypE

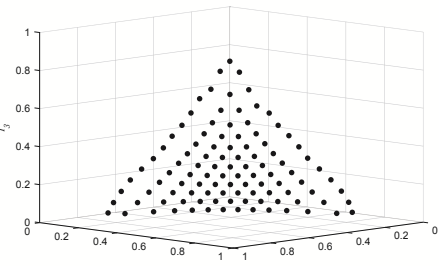

MOEA/D-PBI

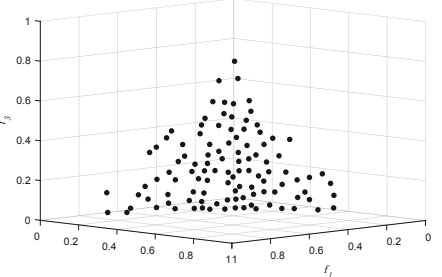

SPEA2+SDE

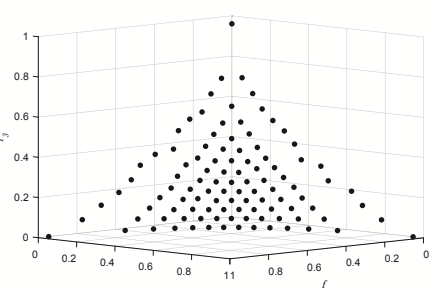

RVEA

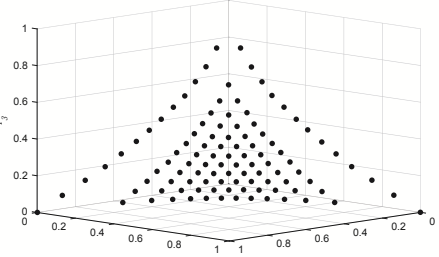

NSGA-III

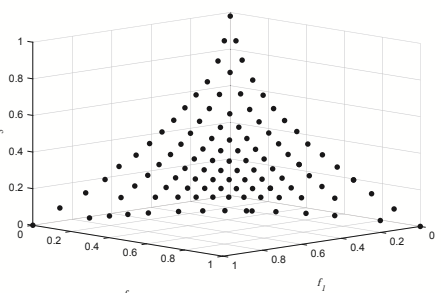

ARMA

Figure 6: Obtained solutions by HypE, SPEA2+SDE, NSGA-III, MOEA/D-PBI, RVEA and ARMA for the convex Pareto-optimal problem CDTLZ2 on 3-objectives.

The WFG3 and DTLZ1 are linear problems. According to Table III, all compared algorithms maintained a good distribution and convergence on the DTLZ1 problem. Despite that, the ARMA had more advantages than RVEA, MOEA/D-PBI and NSGA-III on all objectives. However, the ARMA lost its advantage on WFG3 except on 3 targets. The other algorithms, NSGA-III, PBI and HypE, to a certain tried to a better HV metric for the 2-, and 5-objectives WFG3 problem.

- Discontinuous Pareto Front 


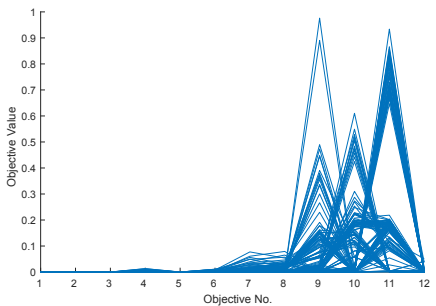

HypE

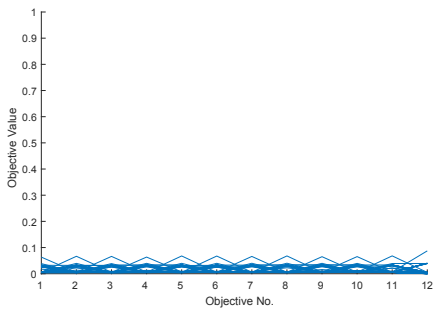

MOEA/D-PBI

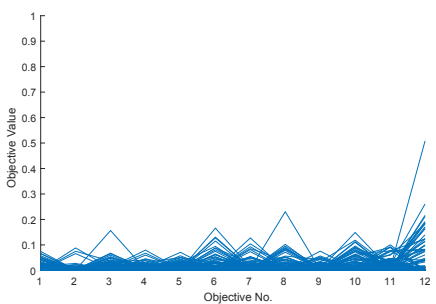

SPEA2+SDE

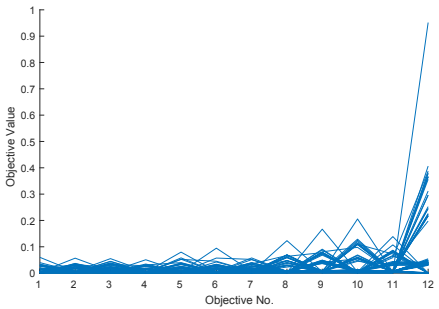

RVEA

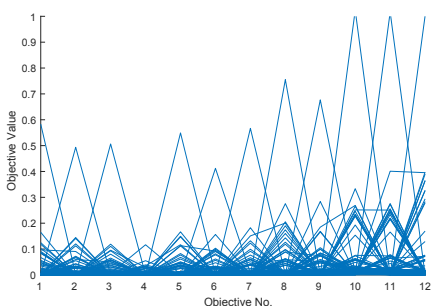

NSGA-III

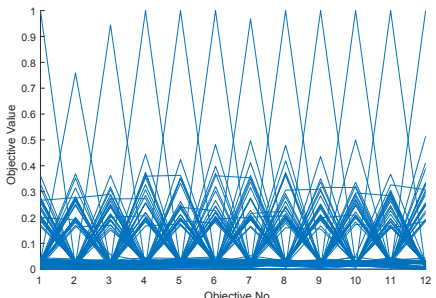

ARMA

Figure 7: Obtained solutions by HypE, SPEA2+SDE, NSGA-III, MOEA/D-PBI, RVEA and ARMA for the convex Pareto-optimal problem CDTLZ2 on 12-objectives.

The WFG2 is a non-continuous, convex PF test problems. As can be clearly observed, the HV values in the table obtained by the ARMA and RVEA were better overall than NSGA-III and MOEA/D-PBI, although they were not competitive with SPEA2+SDE. Indeed, HypE performed best in 5-objectives instances.

- Degenerate Pareto Front

The DTLZ5 and DTLZ6 are designed to measure the convergence ability of MOEA to a curved PF. In fact, the ARMA, like other pre-defined reference point algorithms, cannot fully satisfy degenerate problems. One major reason behind the failure can be attributed to the sharp PF of MOPs. Compared to the MOEA reference-based, nonreference-based MOEA, SPEA2+SDE is more suitable for this category in the experiment. As can be seen in Table III, SPEA2+SDE showed a clearer advantage than referencebased MOEA in 2-, 3- and 5-objective DTLZ5 instances. Although the MOEA/D-PBI performed better than SDE and HypE, SDE and HypE were the most competitive overall in DTLZ5 and DTLZ6 problems.

- Mixed Pareto Front

The WFG1 is a mixed, biased test problem, which poses a huge challenge to MOEA in maintaining diversity. According to the HV from the Table III, SDE performs better than other algorithms. Although the ARMA is only slightly superior to RVEA and HypE, it performed significantly better than NSGA-III and MOEA/D-PBI. 


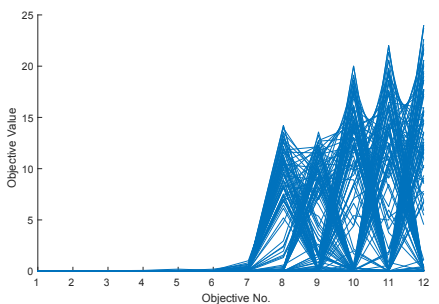

HypE

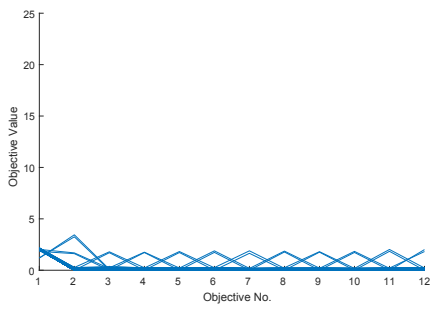

MOEA/D-PBI

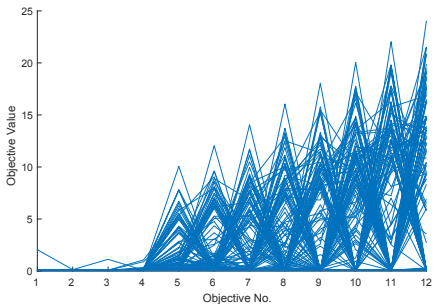

SPEA2+SDE

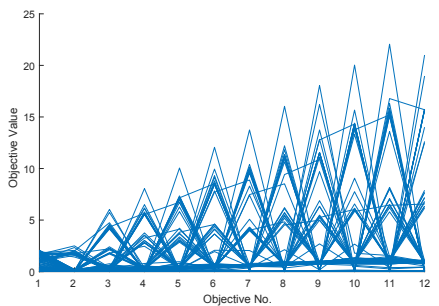

RVEA

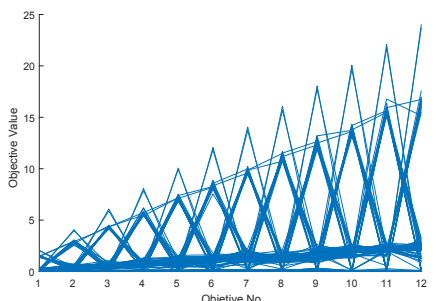

NSGA-III

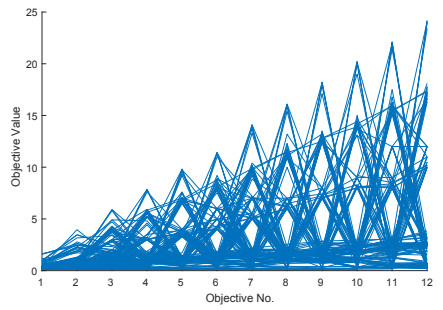

ARMA

Figure 8: Obtained solutions by HypE, SPEA2+SDE, NSGA-III, MOEA/D-PBI, RVEA and ARMA for the concave Pareto-optimal problem WFG4 on 12-objectives.

- Result Summary

To summarize, the ARMA generally outperformed HypE, SPEA2+SDE, NSGA-III, MOEA/D-PBI, and RVEA in terms of convex and concave problems. The ARMA had a better HV value in 30 out of the 50 test instances for the two categories. Unfortunately, the reference point-based ARMA, RVEA, MOEA/D and NSGA-III also failed to maintain their better performance in the discontinuous, degenerate and mixed categories.

\section{Discussion}

One important issue in the reference point adaptation process of the ARMA framework is the setting of parameter $\phi$. The $\phi$ argument directly uses the Euclidean distance to control the convex (or concave) degree of the reference point hyperplane. A large $\phi$ will result in a large degree of convex $\mathrm{PF}$ (or concave $\mathrm{PF}$ ). Therefore, it is critical for the ARMA to set a suitable $\phi$ value in the reference point adaptation process.

In order to verify the influence of different values of $\phi$ in the ARMA, we conducted experiments to analyze its performance on different classifications of $\phi$. To observe the pure effect of $\phi$, other conditions in the algorithm were set the same. The $\phi$ is between $0-1$, and we chose $\phi=0.6,0.65,0.7,0.75,0.8,0.85,0.9$, 0.95 and 1. DTLZ2 was selected as the test problem for $\phi$ with varying number of objectives, and HV metric was normalized to 0-10 for display in Fig. 9. 

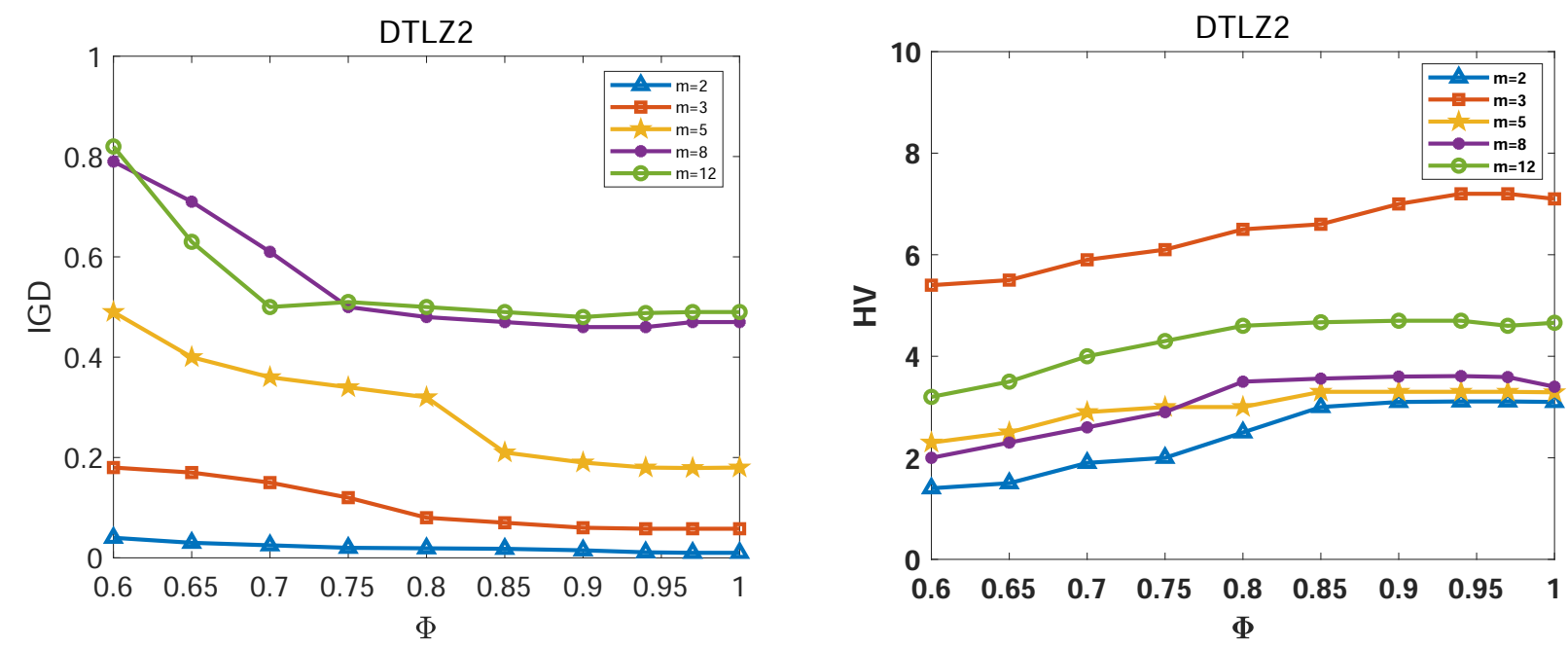

Figure 9: Examination of the influence of $\phi$ on IGD and HV of ARMA for DTLZ2 problems with varying number of objectives $m$.

Fig. 9 presents the performance of the ARMA with various parameter $\phi$ on DTLZ2 problems in terms of average IGD and average HV. As can be seen from Fig. 9, setting $\phi$ to 0.6-0.9 leads to the worst performance according to the IGD metric. The ARMA's better performance was obtained with setting $\phi$ to $0.9-1$, and $\phi=0.95$ was the best parameter having the best overall performance among the experiments. According to this method, the IGD indicator will become better gradually with an increase of the $\phi$ value. This phenomenon indicates that there may be better performance when the reference points have similar PF characteristics. The performance of the ARMA on most of the problem instances is robust as $\phi>0.9$.

\section{CONCLUSION}

In this paper, an alternative has been proposed based on the decomposition-based strategy, termed ARMA, to deal with MOPs with various properties. In the ARMA, a reference point adjustment strategy is used to improve the balance between the convergence and diversity of the solutions during the evolutionary process. The adjustment strategy adjusts the relative position of the reference point based on its neighbor solutions at each generation. Additionally, a new Fitness function maintains the elite individual in the environmental selection by using proportion and angle. The empirical results demonstrate that the proposed ARMA outperforms five representative MOEAs on problems with six various complex characteristics. The results indicate the ARMA applied to the concave and convex problems is more effective than its competitor.

Although the ARMA performed well in the test instances considered in this paper, it also needs to be examined on intermittent and degenerative problems. The reference-point-based algorithm is still in its infancy, and it has several problems that need to be solved. Therefore, these will be addressed in future 
work.

\section{Acknowledgements}

The authors wish to thank the support of the National Natural Science Foundation of China (Grant No. 61876164, 61673331, 61772178), the Education Department Major Project of Hunan Province (Grant No.17A212), the Science and Technology Plan Project of Hunan Province (Grant No.2018TP1036, 2016TP1020), the Provinces and Cities Joint Foundation Project (Grant No.2017JJ4001).

\section{References}

[1] Andr Slflow, Nicole Drechsler, and Rolf Drechsler. Robust multiobjective optimization in high dimensional spaces. In Evolutionary Multi-Criterion Optimization, International Conference, Emo 2007, Matsushima, Japan, March 5-8, 2007, Proceedings, pages 715C726, 2007.

[2] A. Mohammadi and H. Asadi and S. Mohamed and K. Nelson and S. Nahavandi. OpenGA, a C++ Genetic Algorithm Library. 2017 IEEE International Conference on Systems, Man, and Cybernetics, $0(0): 2051-2056,2017$

[3] Alsheddy, \& Abdullah. (2018). A penalty-based multi-objectivization approach for single objective optimization. Information Sciences, 442-443, 1-17.

[4] Anne Auger, Johannes Bader, Dimo Brockhoff, and Eckart Zitzler. Theory of the hypervolume indicator: optimal -distributions and the choice of the reference point. In FOGA 09: Proceedings of the tenth ACM SIGEVO workshop on Foundations of genetic algorithms, pages 87C102, 2009.

[5] Antonio LOpez, Carlos A. Coello Coello, Akira Oyama, and Kozo Fujii. An alternative preference relation to deal with many-objective optimization problems. 7811:291C306, 2013.

[6] Bai, Y. , Qi, Z. , Zhihai, W. , \& Andrew, Z. J. . (2018). Adaptive decomposition-based evolutionary approach for multiobjective sparse reconstruction. Information Sciences, 462, 141-159.

[7] Bingdong Li, Jinlong Li, Ke Tang, and Xin Yao. Many-objective evolutionary algorithms: A survey. Acm Computing Surveys, 48(1):13, 2015.

[8] Cheraghchi, F. , Abualhaol, I. , Falcon, R. , Abielmona, R. , Raahemi, B. , \& Petriu, E. . (2018). Modeling the speed-based vessel schedule recovery problem using evolutionary multiobjective optimization. INFORMATION SCIENCES. 
[9] Chong, Z. , Guangming, D. , Cuijun, Z. , Xiangping, L. , \& Ke, M. . (2018). Entropy based evolutionary algorithm with adaptive reference points for many-objective optimization problems. Information Sciences, S0020025518305255-.

[10] Christian Igel, Nikolaus Hansen, and Stefan Roth. Covariance Matrix Adaptation for Multi-objective Optimization. MIT Press, 2007.

[11] David W. Corne and Joshua D. Knowles. Techniques for highly multiobjective optimisation: some nondominated points are better than others. In Conference on Genetic and Evolutionary Computation, pages 773C780, 2007.

[12] David W. Corne, Nick R. Jerram, Joshua D. Knowles, and Martin J. Oates. Pesa-ii: region-based selection in evolutionary multiobjective optimization. In Conference on Genetic and Evolutionary Computation, pages 283C290, 2001.

[13] Eckart Zitzler and Lothar Thiele. Multiobjective evolutionary algorithms: a comparative case study and the strength pareto approach. IEEE Transactions on Evolutionary Computation, 3(4):257C271, 1999.

[14] Eckart Zitzler and Simon Knzli. Indicator-based selection in multiobjective search. In International Conference on Parallel Problem Solving from Nature, pages 832C842, 2004.

[15] Eckart Zitzler, Kalyanmoy Deb, and Lothar Thiele. Comparison of multiobjective evolutionary algorithms: Empirical results. Evol Comput., 8(2):173C195, 2000.

[16] Eckart Zitzler, Marco Laumanns, and Lothar Thiele. Spea2: Improving the strength pareto evolutionary algorithm for multiobjective optimization. In Evolutionary Methods for Design, Optimization and Control with Applications To Industrial Problems. Proceedings of the Eurogen2001. Athens. Greece, September, 2001.

[17] Fangqing Gu, H. L. Liu, and Kay Chen Tan. A multiobjective evolutionary algorithm using dynamic weight design method. International Journal of Innovative Computing Information Control Ijicic, 8(5):3677C 3688, 2011.

[18] Frank Wilcoxon. Individual comparisons by ranking methods. Biometrics Bulletin, 1(6):80C83, 1945.

[19] Gema, B. O. , Sancho, S. S. , \& David, C. . (2018). A multi-objective genetic algorithm for overlapping community detection based on edge encoding. Information Sciences, 462, 290-314.

[20] Guo-Zhong F , Yan-Feng L , Ye T , et al. An interactive preference-based evolutionary algorithm for multi-criteria satisficing optimization[J]. Journal of Intelligent \& Fuzzy Systems, 2018, 34(4):25032511. 
[21] Hai Lin Liu, Fangqing Gu, and Qingfu Zhang. Decomposition of a multiobjective optimization problem into a number of simple multiobjective subproblems. IEEE Transactions on Evolutionary Computation, 18(3):450C455, 2014.

[22] Halim Z , Baig A R , Zafar K . Evolutionary Search in the Space of Rules for Creation of New Two-Player Board Games[J]. International Journal on Artificial Intelligence Tools, 2014, 23(02):1350028.

[23] Halim Z , Muhammad T . Quantifying and Optimizing Visualization: An Evolutionary Computing-Based Approach[J]. Information Sciences, 2017, 385:284C313.

[24] Halim Z , Uzma, . Optimizing the minimum spanning tree-based extracted clusters using evolution strategy[J]. Cluster Computing, 2017.

[25] Halim Z , Waqas M , Hussain S F . Clustering large probabilistic graphs using multi-population evolutionary algorithm[J]. Information Sciences, 2015, 317:78-95

[26] Hancer, E. , Xue, B. , Zhang, M. , Karaboga, D. , \& Akay, B. . (2017). Pareto front feature selection based on artificial bee colony optimization. Information Sciences, S0020025516312609.

[27] I Das and J. E. Dennis. Normal-boundary intersection: A new method for generating the pareto surface in nonlinear multicriteria optimization problems. Siam Journal on Optimization, 8(3):631C657, 1998.

[28] J Vesanto and E Alhoniemi. Clustering of the self-organizing map. IEEE Transactions on Neural Networks, 11(3):586, 2002.

[29] JAIN, Himanshu, Kalyanmoy. An evolutionary many-objective optimization algorithm using referencepoint based nondominated sorting approach, Part II: Handling constraints and extending to an adaptive approach[J]. IEEE Transactions on Evolutionary Computation, 2014, 18(4):602-622.

[30] Jaszkiewicz. 2002. On the performance of multiple-objective genetic local search on the 0/1 knapsack problem-A comparative experiment. IEEE Transactions on Evolutionary Computation 6, 4, 402C412.

[31] Jess Garcia, Antonio Berlanga, and Jos Manuel Molina Lpez. Effective evolutionary algorithms for manyspecifications attainment: Application to air traffic control tracking filters. IEEE Transactions on Evolutionary Computation, 13(1):151C168, 2009.

[32] Johannes Bader and Eckart Zitzler. Hype: An algorithm for fast hypervolume-based many-objective optimization. MIT Press, 2011.

[33] Kalyanmoy Deb and Himanshu Jain. An evolutionary many-objective optimization algorithm using reference-point-based nondominated sorting approach, part i: Solving problems with box constraints. IEEE Transactions on Evolutionary Computation, 18(4):577C601, 2014. 
[34] Kalyanmoy Deb, Amrit Pratap, Sameer Agarwal, and T Meyarivan. A fast and elitist multiobjective genetic algorithm: NSGA-II. IEEE Transactions Evolutionary Computation, 6(2):182C197, 2002.

[35] Kalyanmoy Deb, Lothar Thiele, Marco Laumanns, and Eckart Zitzler. Scalable Test Problems for Evolutionary Multiobjective Optimization. 2005.

[36] Karl Bringmann and Tobias Friedrich. Dont be greedy when calculating hypervolume contributions. pages 103C112, 2009.

[37] Ke Li, Kalyanmoy Deb, Qingfu Zhang, and Sam Kwong. An evolutionary many-objective optimization algorithm based on dominance and decomposition. IEEE Transactions on Evolutionary Computation, 19(5):694C716, 2015.

[38] Khan, B. , Hanoun, S. , Johnstone, M. , Lim, C. P. , Creighton, D. , \& Nahavandi, S. . (2018). A scalarization-based dominance evolutionary algorithm for many-objective optimization. Information Sciences.

[39] K Ikeda, H Kita, and S Kobayashi. Failure of pareto-based moeas: does non-dominated really mean near to optimal? In Evolutionary Computation, 2001. Proceedings of the 2001 Congress on, pages 957C 962 vol. 2, 2001.

[40] Li B , Li J , Tang K, et al. Many-Objective Evolutionary Algorithms: A Survey[J]. Acm Computing Surveys, 2015, 48(1):1-35.

[41] Liuwei Fu, Juan Zou, Shengxiang Yang, Ruan Gan, Zhongwei Ma, and Jinhua Zheng. A proportionbased selection scheme for multi-objective optimization. pages 1C7, 2017.

[42] Lyndon While, Lucas Bradstreet, and Luigi Barone. A fast way of calculating exact hypervolumes. IEEE Transactions on Evolutionary Computation, 16(1):86C95, 2012.

[43] M Farina and P Amato. A fuzzy definition of optimality for manycriteria optimization problems. IEEE Transactions on Systems Man and Cybernetics - Part A Systems and Humans, 34(3):315C326, 2004.

[44] Manikanth Mohan, Manikanth Mohan, and Shikhar Mishra. Evaluating the epsi; -Domination Based Multi-Objective Evolutionary Algorithm for a Quick Computation of Pareto-Optimal Solutions. MIT Press, 2005

[45] Marco Laumanns, Lothar Thiele, Kalyanmoy Deb, and Eckart Zitzler. Combining convergence and diversity in evolutionary multi-objective optimization. pages 263 C 282, 2002.

[46] Miqing Li, Shengxiang Yang, and Xiaohui Liu. Pareto or non-pareto: Bi-criterion evolution in multiobjective optimization. In The IEEE International Conference on Fuzzy Systems, pages 1319C1322, 2001. 
[47] Miqing Li, Shengxiang Yang, and Xiaohui Liu. Shift-based density estimation for pareto-based algorithms in many-objective optimization. IEEE Transactions on Evolutionary Computation, 18(3):348C365, 2014.

[48] Nicola Beume, Boris Naujoks, and Michael Emmerich. Sms-emoa: Multiobjective selection based on dominated hypervolume. European Journal of Operational Research, 181(3):1653C1669, 2007.

[49] Nicole Drechsler, Rolf Drechsler, and Bernd Becker. Multi-objective optimisation based on relation favour. Lecture Notes in Computer Science, 1993(1993):154C166, 2001.

[50] Pan, A. , Wang, L. , Guo, W. , \& Wu, Q. . (2018). A diversity enhanced multiobjective particle swarm optimization. Information Sciences, S0020025518300549.

[51] Peter J. Fleming, Robin C. Purshouse, and Robert J. Lygoe. Manyobjective optimization: An engineering design perspective. In International Conference on Evolutionary Multi-Criterion, pages 14C32, 2005.

[52] Qingfu Zhang and Hui Li. MOEA/D: A Multiobjective Evolutionary Algorithm Based on Decomposition. IEEE Press, 2007.

[53] R. C. Purshouse and P. J. Fleming. Evolutionary many-objective optimisation: an exploratory analysis. In Evolutionary Computation, 2003. CEC 03. The 2003 Congress on, pages 2066C2073 Vol.3, 2003.

[54] Ran Cheng, Yaochu Jin, Markus Olhofer, and Bernhard Sendhoff. A reference vector guided evolutionary algorithm for many-objective optimization. IEEE Transactions on Evolutionary Computation, 20(5):773C 791, 2016.

[55] S. Huband, P. Hingston, L. Barone, and L. While. A review of multiobjective test problems and a scalable test problem toolkit. IEEE Transactions on Evolutionary Computation, 10(5):477C506, 2006.

[56] Sengupta, R. , \& Saha, S. . (2018). Reference point based archived many objective simulated annealing. Information Sciences, S0020025518303669.

[57] Shengxiang Yang, Miqing Li, Xiaohui Liu, and Jinhua Zheng. A gridbased evolutionary algorithm for many-objective optimization. IEEE Transactions on Evolutionary Computation, 17(5):721C736, 2013.

[58] Shouyong Jiang and Shengxiang Yang. A strength pareto evolutionary algorithm based on reference direction for multiobjective and many-objective optimization. IEEE Transactions on Evolutionary Computation, 21(3):329C346, 2017.

[59] Tadahiko Murata, Hisao Ishibuchi, and Mitsuo Gen. Specification of Genetic Search Directions in Cellular Multi-objective Genetic Algorithms. Springer Berlin Heidelberg, 2001. 
[60] Tian Y, Cheng R, Zhang X, et al. An Indicator Based Multi-Objective Evolutionary Algorithm with Reference Point Adaptation for Better Versatility[J]. IEEE Transactions on Evolutionary Computation, 2017, PP(99):1-1.

[61] Wong, C. S. Y. , Al-Dujaili, A. , Suresh, S. , \& Sundararajan, N. . (2018). Pareto-aware strategies for faster convergence in multi-objective multi-scale search optimization. Information Sciences, S0020025518303220.

[62] Xie, Z. , Sun, J. , Palade, V. , Wang, S. , \& Liu, Y. . (2015). Evolutionary sampling: a novel way of machine learning within a probabilistic framework. Information Sciences, 299, 262-282.

[63] Y. Liu, N. Zhu and K. Li et al., An angle dominance criterion for evolutionary many-objective optimization, Information Sciences, 10.1016/j.ins.12.078

[64] Yuan Yuan, Hua Xu, Bo Wang, and Xin Yao. A new dominance relationbased evolutionary algorithm for many-objective optimization. IEEE Transactions on Evolutionary Computation, 20(1):16C37, 2016.

[65] Zangari, M. , Mendiburu, A. , Santana, R. , \& Pozo, A. . (2017). Multiobjective decomposition-based mallows models estimation of distribution algorithm. a case of study for permutation flowshop scheduling problem. Information Sciences, 397-398, 137-154.

[66] Zhang, Y. , Gong, D. , Sun, J. , \& Qu, B. . (0). A decomposition-based archiving approach for multiobjective evolutionary optimization. INFORMATION SCIENCES.

[67] Zhao, H. , Zhang, C. , Zhang, B. , Duan, P. , \& Yang, Y. . (2018). Decomposition-based sub-problem optimal solution updating direction-guided evolutionary many-objective algorithm. Information Sciences, S0020025518301932.

[68] Zhu, Q. , Lin, Q. , Du, Z. , Liang, Z. , Wang, W. , \& Zhu, Z. , et al. (2016). A novel adaptive hybrid crossover operator for multiobjective evolutionary algorithm. Information Sciences, S0020025516000803.

[69] Zou, J. , Fu, L. , Yang, S. , Zheng, J. , Yu, G. , \& Hu, Y. . (2018). A many-objective evolutionary algorithm based on rotated grid. Applied Soft Computing, S1568494618300905.

[70] Gan, R., Guo, Y., Zheng, J., Zou, J., Yang, S. (2017). The effect of diversity maintenance on prediction in dynamic multi-objective optimization. Applied Soft Computing, 58, 631-647.

[71] Zou, J., Zhang, Y., Yang, S., Yuan, L., Zheng, J. (2018). Adaptive neighborhood selection for manyobjective optimization problems. Applied Soft Computing, 64, 186-198.

[72] Li, Q., Zou, J., Yang, S., Zheng, J., Gan, R. (2018). A predictive strategy based on special points for evolutionary dynamic multi-objective optimization. Soft Computing(1), 1-17. 